\title{
Evolución clínica, funcional y radiológica de pacientes trasplantados pulmonares con síndrome de bronquiolitis obliterante y disfunción de injerto pulmonar restrictivo
}

\author{
M. TERESA PARADA C.*, CONSTANZA RIFFO S.**, RAQUEL RETAMAL C.** y JOEL MELO T.*
}

Clinical, functional and radiological evolution of pulmonary transplant patients with bronchiolitis obliterans syndrome and restrictive graft dysfunction

The main long-term complication in lung transplant patients is chronic graft dysfunction identified as bronchiolitis obliterans, and there is a new pattern called Restrictive Graft Dysfunction. Objective: To evaluate spirometric, radiological and clinical follow-up among patients with bronchiolitis obliterans syndrome (BOS) and Restrictive Allograft Syndrome (RAS) after lung transplantation. Methodology: Lung transplant recipients' clinical records were reviewed from 1999 to 2017. We carried out a follow up of spirometry, chest tomography imaging and associated factors: cytomegalovirus $(C M V)$ infection, gastroesophageal reflux (GER) and episodes of acute rejection. Survival was analyzed by Kaplan Meier. Results: Out of 88 lung transplant patients, 40 developed chronic graft dysfunction: $31(80 \%)$ presented BOS and 9 (20\%) had RAS. Mean age: 47 yro. in BOS and 46 yr. o. in RAS. Lung fibrosis was the primary pathology predominant in both conditions. In BOS were reported 14 episodes of acute rejection (50\%), CMV infection in $18 \%$ and active GER in $26 \%$. In RAS there were 5 episodes of acute rejection (62\%), CMV infection in $13 \%$ and active GER in $67 \%(p=0.02)$. VEF $F_{1}$ follow-up at 1-2-4-5 years averaged 67, 65, 60 and 8\% of reference value in BOS and 61, 65, 62 and 45\% in $R A S$ respectively. CT scans showed hyperinflation in BOS and upper pleuropulmonary fibrosis in RAS. $B O S$ survival time was 96.9 months versus 65.6 months in $R A S(p=0.06)$. Conclusions: Restrictive dysfunction presented a lower survival rate than BOS. GER was associated with restrictive rejection. Chest tomography differs in both types of chronic rejection.

Key words: Lung transplantation, Bronchiolitis Obliterans, Allograft, Cytomegalovirus, Gastroesophageal reflux.

\section{Resumen}

La principal complicación a largo plazo en trasplantados de pulmón es la disfunción crónica de injerto identificado como bronquiolitis obliterante, existiendo un nuevo patrón denominado Disfunción de Injerto Restrictivo. Objetivo: Evaluar seguimiento espirométrico, radiológico y clínico entre pacientes con síndrome de bronquiolitis obliterante (SBO) y Disfunción de Injerto Restrictivo (DIR) post trasplante pulmonar. Metodología: Se revisaron registros clínicos de trasplantados pulmonares desde 1999 hasta 2017. Se efectuó seguimiento espirométrico e imágenes por tomografía de tórax y factores asociados: infección por Citomegalovirus(CMV), reflujo gastro-esofágico (RGE) y episodios de rechazo agudo. Se analizó sobrevida por Kaplan Meier. Resultados: De 88 pacientes trasplantados de pulmón, 40 desarrollaron disfunción crónica de injerto: 31 (80\%) presentaron SBO y 9 (20\%) tuvieron DIR. Edad promedio: 47 años en SBO y 46 años en DIR. Siendo fibrosis pulmonar la patología basal predominante en ambos. En SBO se consignaron 14 episodios de rechazo agudo (50\%), infección por CMV en $18 \%$ y RGE activo en 26\%. En la serie DIR hubo 5 episodios de rechazo agudo (62\%),

* Centro de Enfermedades Respiratorias, Clínica Las Condes.

** Becada Hospital Dipreca: Dirección de Previsión de Carabineros de Chile. 
$13 \%$ de infección por CMV y 67\% de RGE activo $6(p=0,02)$. En el seguimiento a 1-2-4-5 años el promedio del $V E F_{1}$ en $S B O$ fue: 67,3 , 65, 60 y 48\% del valor predicho y en DIR fue 61, 65, 62 y 45\% respectivamente. Las imágenes tomográficas en SBO mostraron: hiperinflación y en DIR: fibrosis pleuropulmonar superior. La sobrevida fue de 96,9 meses en SBO y 65,6 meses en DIR ( $p=0,06)$. Conclusiones: La disfunción restrictiva presentó menor sobrevida que $S B O$. RGE se asoció a rechazo restrictivo. La tomografía de tórax difiere en ambos tipos de rechazo crónico.

Palabras clave: Trasplante pulmonar, Bronquiolitis Obliterante, homoinjerto, Citomegalovirus, Reflujo gastroesofágico.

\section{Introduccion}

El trasplante de pulmón es una alternativa terapéutica en un grupo seleccionado de pacientes aquejados de una enfermedad respiratoria terminal, tal como Fibrosis pulmonar, Fibrosis quística o Enfermedad pulmonar Obstructiva crónica (EPOC). La sobrevida del trasplante pulmonar es menor a la de otros injertos de órganos sólidos, alcanzando alrededor de $50-55 \%$ a 5 años ${ }^{1}$. La mayor limitación a largo plazo la constituye la disfunción crónica del injerto, reconocida como Síndrome de Bronquiolitis Obliterante (SBO), caracterizada por una caída del $\mathrm{VEF}_{1}$ (volumen espiratorio forzado en el $1^{\mathrm{er}} \mathrm{s}$ ) progresiva e irreversible en la espirometría y que no puede ser atribuido a otra causa. El estudio de imágenes tomográficas revela atrapamiento aéreo y atenuación en mosaico y que en caso de realizar estudio histopatológico el sello es la bronquiolitis obliterativa de vía aérea pequeña ${ }^{2}$. En la actualidad se ha observado una forma diferente de disfunción crónica de injerto, con un componente restrictivo y acompañado de infiltrados pleuropulmonares periféricos con aspecto de fibrosis predominante en lóbulos superiores y que se ha definido como Disfunción de Injerto Restrictivo (DIR), el que determinaría una peor sobrevida en los pacientes $^{3}$.

El objetivo de este estudio es evaluar seguimiento espirométrico, radiológico y sobrevida en pacientes con disfunción crónica de injerto de tipo SBO y DIR posterior a 6 meses del trasplante pulmonar.

\section{Pacientes y Método}

Se revisaron registros clínicos de pacientes trasplantados de pulmón en Clínica Las Condes desde 1999 hasta abril de 2017. Se consideraron los pacientes con seguimiento mayor a 6 meses y que desarrollaron signos de disfunción crónica de injerto. Se analizaron datos demográficos, patología de base, seguimiento espirométrico, distancia caminada en $6 \mathrm{~min}$, imágenes por tomografía de tórax. El estudio broncoscópico con lavado broncoalveolar (LBA) y biopsia transbronquial se realizó solo en pacientes con deterioro rápido y progresivo para descartar otras etiologías como lesión de vía aérea, rechazo agudo o infección.

El esquema de inmunosupresión se ha mantenido sin cambios respecto a la inducción con basiliximab y terapia de mantención triasociada con tacrolimus, micofenolato o azatioprina $\mathrm{y}$ esteroides

\section{Definiciones de tipo de disfunción crónica de injerto}

SBO: Se consideró a la caída de $\mathrm{VEF}_{1}<80 \%$ del promedio de 2 mejores del post operatorio y persistente en 3 meses, descartado infección aguda o lesión de vía aérea, acompañado de imagen de atrapamiento aéreo en tomografía de tórax.

DIR: Caída persistente del $\mathrm{VEF}_{1}<80 \%$ en la espirometría, acompañado de reducción de capacidad vital forzada (CVF), descartado proceso infeccioso y asociado a imagen tomográfica sugerente de fibroelastosis pleuropulmonar.

Se registraron factores asociados como: infección por Citomegalovirus (CMV), reflujo gastro-esofágico sintomático (RGE), número de episodios de rechazo precoz $(<12$ meses $)$ y desarrollo de anticuerpos "de novo".

Se realizó test de Mann-Whitney para muestras independientes, para determinar si existían diferencias significativas entre ambos rechazos en cuanto a la edad, presencia de rechazo agudo, serología e infección por CMV y RGE. Un valor de $\mathrm{p} \leq 0,05$ fue considerado significativo.

Se analizó sobrevida según Kaplan-Meier en ambas series SBO y DIR.

\section{Resultados}

Entre el año 2009 y abril de 2017 se realizaron 88 trasplantes pulmonares en Clínica Las Condes y 68 completaron seguimiento mayor a 6 meses, de los cuales 40 desarrollaron disfunción crónica de injerto, 31 (80\%) de tipo SBO y 9 (20\%) DIR.

La edad promedio de trasplante fue de 47 
años (rango: 16-70) en la serie SBO y 46 años (rango: 13-71) en la serie DIR. La patología de base predominante en ambas series de pacientes fue la fibrosis pulmonar idiopática $61 \%$ y la técnica monopulmonar se realizó en el $71 \%$ en la serie SBO y en el $56 \%$ de los pacientes de la serie DIR. La presencia de sensibilización pretrasplante fue baja en ambas series $(\mathrm{PRA}<10 \%)$ (Tabla 1). En nuestra revisión la presencia de Disfunción Primaria de Injerto (DPI) ocurrió en 6 de 31 pacientes en la serie SBO 4 de ellas fueron severas requiriendo uso de circulación extracorpórea (ECMO) en tres pacientes y obligó a ventilación mecánica por un período mayor a 7 días. En la serie DIR hubo 3 episodios de DPI en 9 pacientes y dos en rango severo que requirieron de apoyo con ECMO.

En la serie SBO se consignaron 16 episodios de rechazo agudo precoz en 14 pacientes $(51 \%)$, por clasificación de tipo leve A2 en el $63 \%, 25 \%$ moderado A3 y $12 \%$ severo A4, el tratamiento fue con pulsos de metilprednisolona. Dos pacientes desarrollaron rechazo humoral y fueron tratadas con plasmaféresis y gamaglobulina, 3 desarrrollaron anticuerpos "de novo" a los 4-14 y 60 meses. La infección por CMV ocurrió en 16\% en forma previa a la aparición de disfunción de injerto, destacando serología negativa a CMV al momento del trasplante en 4 de 5 pacientes que presentaron infección. El antecedente de RGE activo se obtuvo en 8 pacientes $26 \%$, cuatro de ellos confirmado por impedanciometría y uno fue sometido a cirugía laparoscópica para calibrar el esfínter gastroesofágico.

En la serie DIR de nueve pacientes hubo 4 episodios de rechazo precoz (44\%). No se demostró presencia de anticuerpos "de novo". La infección por CMV ocurrió en un paciente solamente y fue posterior al tratamiento del rechazo agudo, y el RGE activo se detectó en un $67 \%$ de los pacientes alcanzando significación estadística con un valor de $p=0,02$. Se puede afirmar que el RGE es un factor de riesgo para el rechazo restrictivo, con un RR: 2,62, p < 0,01. Es de destacar que en este grupo 3 pacientes tenían fibrosis quística como patología de base.

El seguimiento de la función pulmonar por espirometría en las series SOB y DIR se describe según la evolución del $\mathrm{VEF}_{1}$ en la Figura 1. La distancia promedio recorrida en el test de caminata 6 min por los pacientes de ambas series se presenta en la Figura 2.

Las imágenes tomográficas se describen en Figuras 3 y 4, destacando en la serie SBO hiperinflación, atrapamiento aéreo con fenómeno de mosaico (Figura 3) y en la serie DIR: signos de tipo fibroelastosis pleuropulmonar de predominio superior (Figura 4).

El estudio broncoscópico en pacientes con disfunción de injerto crónica se realizó solo si existían infiltrados pulmonares o el deterioro funcional era rápidamente progresivo, para descartar infección o lesión de sutura. En total en ambas series se realizó broncoscopía a 14 pacientes, el

Tabla 1. Características demográficas en rechazo crónico tipo Síndrome de Bronquiolitis Obliterante (SBO) y Disfunción de Injerto Restrictivo (DIR)

\begin{tabular}{|c|c|c|c|c|c|}
\hline & $\operatorname{SBO}(n=31)$ & $\%$ & $\operatorname{DIR}(\mathbf{n}=9)$ & $\%$ & Valor de $\mathbf{p}^{(\mathbf{a})}$ \\
\hline Edad promedio años & 47 & & 46 & & 0,20 \\
\hline $\begin{array}{l}\text { Patología de base: } \\
\text { Fibrosis pulmonar } \\
\text { Fibrosis quística } \\
\text { Silicosis } \\
\text { Otros }\end{array}$ & $\begin{array}{r}20 \\
2 \\
4 \\
5\end{array}$ & $\begin{array}{r}66 \\
6 \\
13 \\
15\end{array}$ & $\begin{array}{l}5 \\
3 \\
1 \\
0\end{array}$ & $\begin{array}{l}56 \\
33 \\
11\end{array}$ & \\
\hline $\begin{array}{l}\text { Trasplante monopulmonar } \\
\text { Trasplante bipulmonar }\end{array}$ & $\begin{array}{r}22 \\
9\end{array}$ & $\begin{array}{l}71 \\
29\end{array}$ & $\begin{array}{l}5 \\
4\end{array}$ & $\begin{array}{l}56 \\
44\end{array}$ & \\
\hline $\mathrm{DPI}^{(\mathrm{b})}$ & 6 & 19 & 3 & 33 & \\
\hline $\begin{array}{l}\text { Rechazo agudo } \\
\text { Serología CMV (+) } \\
\text { Infección a CMV } \\
\text { RGE + }\end{array}$ & $\begin{array}{r}16 \\
22 \\
5 \\
8\end{array}$ & $\begin{array}{l}51 \\
71 \\
16 \\
26\end{array}$ & $\begin{array}{l}4 \\
5 \\
1 \\
6\end{array}$ & $\begin{array}{l}44 \\
56 \\
11 \\
67\end{array}$ & $\begin{array}{l}0,6 \\
0,72 \\
0,01\end{array}$ \\
\hline $\begin{array}{l}\text { PRA }<10^{(\mathrm{c})} \\
\text { Anticuerpos DE }(+)^{(\mathrm{d})}\end{array}$ & $\begin{array}{r}29 \\
3\end{array}$ & $\begin{array}{l}94 \\
10\end{array}$ & $\begin{array}{l}9 \\
0\end{array}$ & 100 & \\
\hline
\end{tabular}

${ }^{(a)}$ Test de Mann-Whitney para muestras independientes; ${ }^{(b)}$ DPI: Disfunción Primaria de Injerto; ${ }^{\left({ }^{c}\right)}$ PRA: grado de sensibilidad a anticuerpos previo al trasplante; (d)Anticuerpos DE: anticuerpos donante específico. 

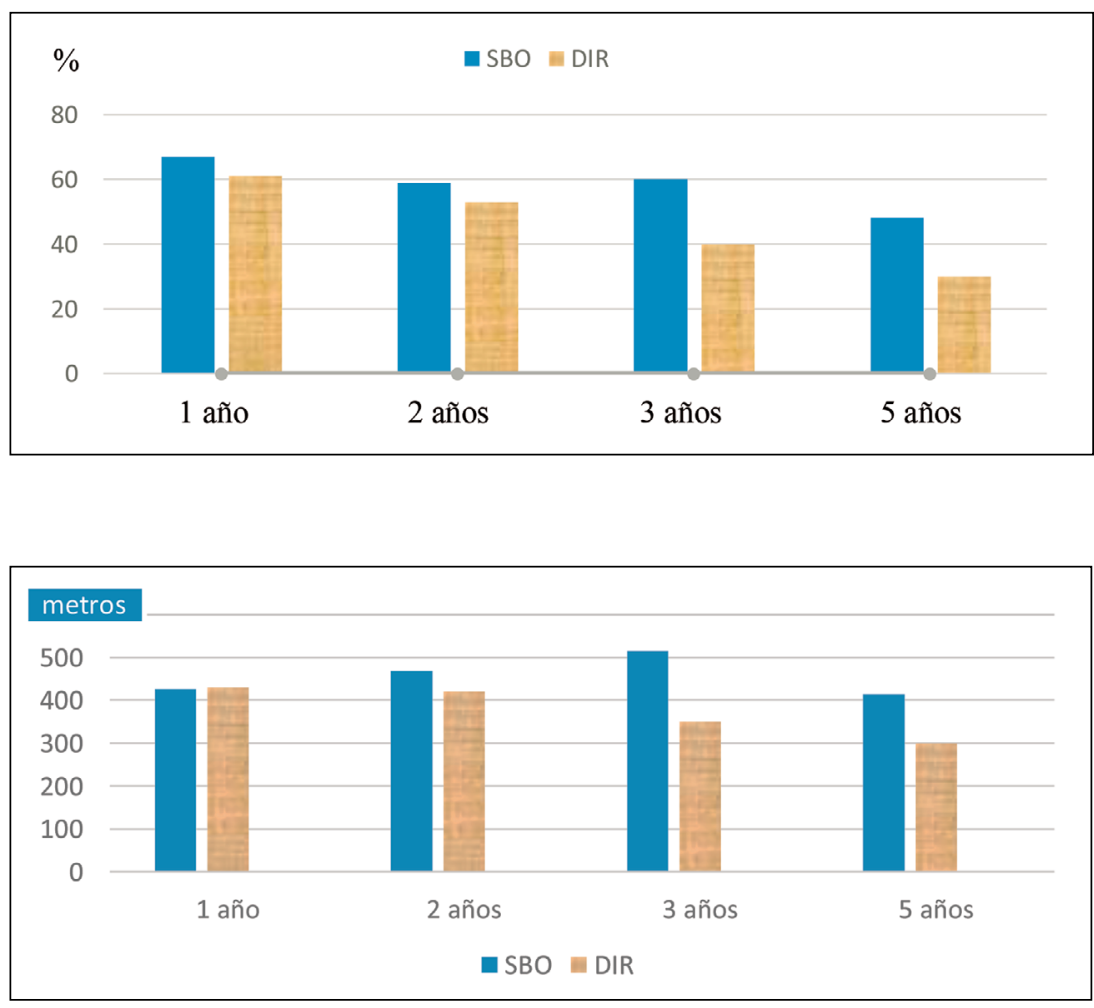

Figura 1. Evolución del $\mathrm{VEF}_{1}$ en rechazo crónico en las series Síndrome de Bronquiolitis Obliterante (SBO) y Disfunción de Injerto Restrictivo (DIR). La altura de las columnas representa el promedio del porcentaje del $\mathrm{VEF}_{1}$ en relación $(\%)$ a su valor de referencia normal en cada serie.

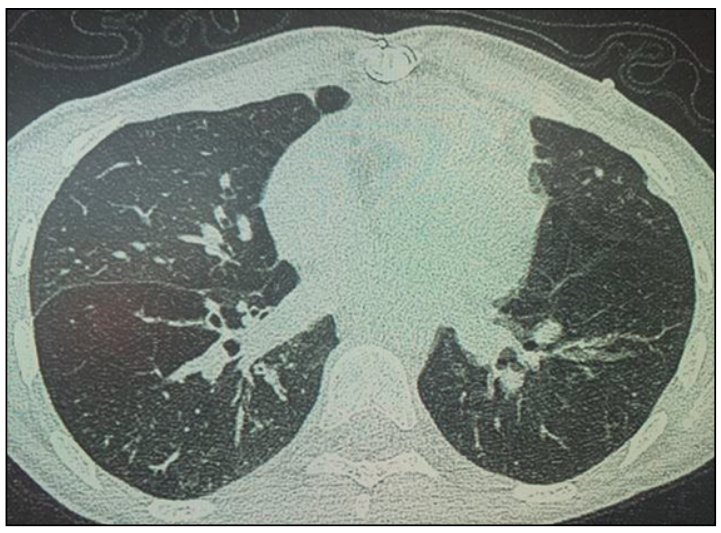

Figura 3. Tomografía de tórax en rechazo crónico tipo Síndrome de Bronquiolitis Obliterante (SBO). La imagen corresponde a un paciente con trasplante pulmonar bilateral y en ella se observa atrapamiento aéreo y atenuación en mosaico.

LBA fue de predominio neutrofílico y la biopsia transbronquial descartó rechazo celular agudo en todos los pacientes.

En la serie SBO de 31 pacientes, 16 fallecieron por esta causa en promedio a los 40 meses post diagnóstico en un rango variable en 4 meses en un paciente rápidamente progresivo, hasta 84 meses en un paciente con declinación lenta de

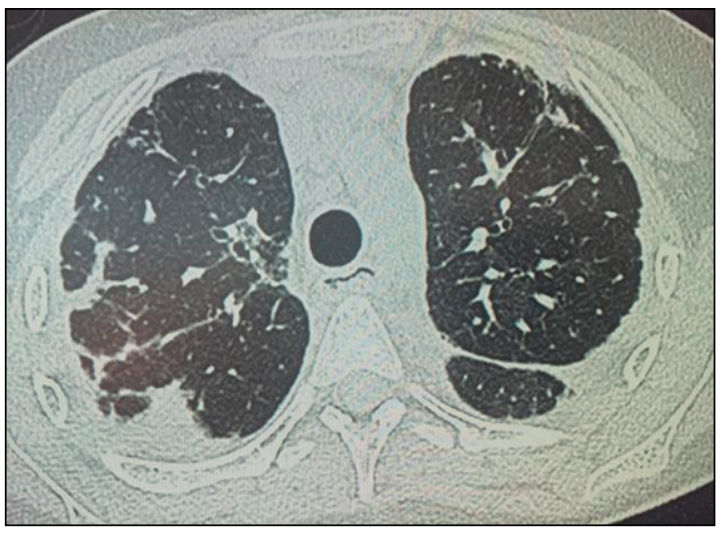

Figura 4. Tomografía de tórax en rechazo crónico tipo Disfunción de Injerto Restrictivo (DIR). La imagen correspone a un paciente con trasplante bipulmonar y en ella se observa fibrosis pleuropulmonar de predominio superior.

su espirometría. Ocho pacientes están vivos y en buenas condiciones y 4 fallecieron por otras causas médicas.

En la serie DIR de los nueve pacientes que lo presentaron 8 fallecieron por deterioro del injerto, en promedio 15 meses después de este diagnóstico con un rango de 5 a 36 meses.

La sobrevida en meses calculada por Kaplan- 


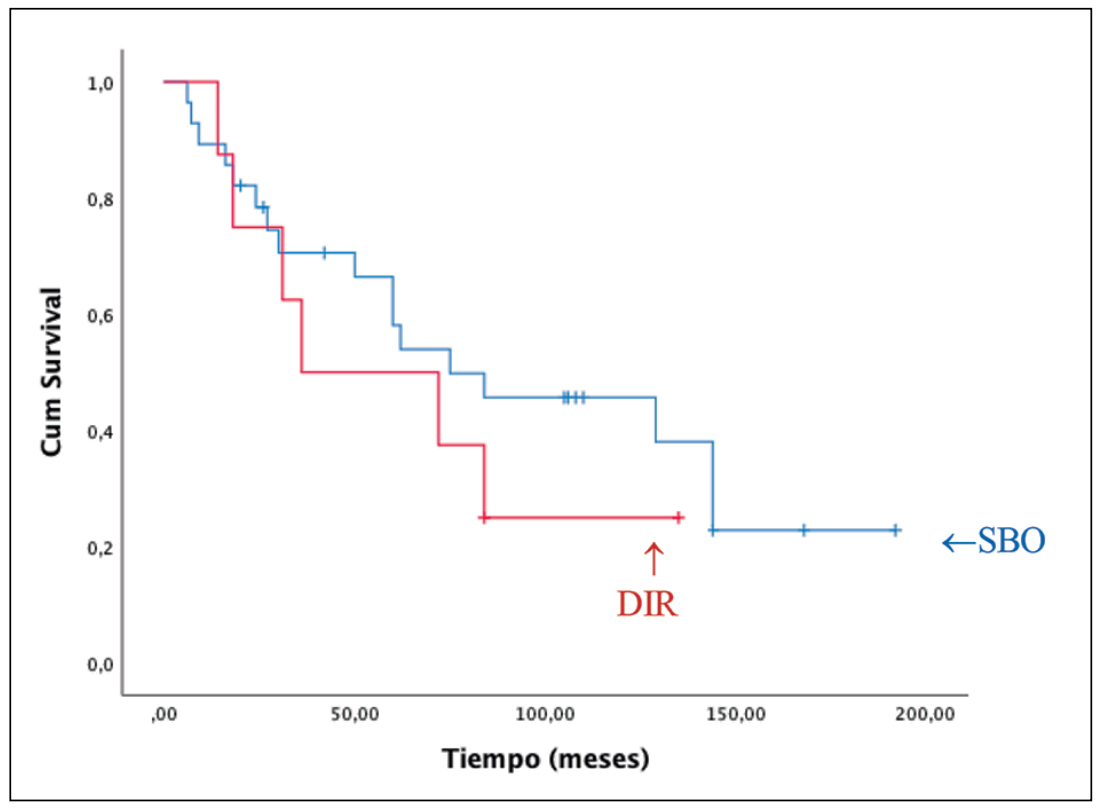

Figura 5. Curvas de sobrevida en rechazo crónico tipo Síndrome de Bronquilitis Obliterante (SBO) y tipo Disfunción de Injerto Restrictivo ( DIR). Cum Survival: Supervivencia global acumulada calculada según Kaplan Meier.
Meier (Figura 5) fue de 96,9 meses en la serie SBO y 65,6 meses en rechazo de injerto restrictivo $(\mathrm{p}=0,06)$.

\section{Discusión}

El trasplante pulmonar constituye una terapia aceptada desde fines de la década de los '80 en pacientes seleccionados aquejados de patología respiratoria en etapa avanzada o terminal. Los resultados a largo plazo se encuentran limitados por el desarrollo de disfunción crónica del injerto, alcanzando alrededor del $50 \%$ a los 5 años posterior al trasplante, y la sobrevida media post diagnóstico de éste es entre 3 y 5 años ${ }^{4}$.

Durante muchos años el SBO, cuyo sello histológico es la bronquiolitis obliterativa de la vía aérea pequeña fue la manifestación clásica de disfunción crónica del injerto, cuyo diagnóstico es principalmente clínico y caracterizado por deterioro progresivo del $\mathrm{VEF}_{1}$ en la espirometría en ausencia de otra causa que lo explique ya sea infecciosa o lesión crónica de vía aérea, y cuya imagen en la tomografía de tórax es el atrapamiento aéreo y atenuación en mosaico. Sin embargo, la definición ha sido cuestionada por la aparición de un fenotipo de disfunción crónica diferente, con componente restrictivo funcional y destacando la aparición de infiltrados pleuropulmonares periféricos de tipo fibróticos y que se ha denominado DIR, el cual constituiría un peor pronóstico para el paciente ${ }^{5}$. Existen escasas publicaciones en la literatura médica comparando la evolución de ambas formas de deterioro del injerto, en búsqueda de factores predictores y de riesgo a desarrollar uno $\mathrm{u}$ otro fenotipo ${ }^{6}$.

En nuestro análisis de seguimiento en pacientes que desarrollaron disfunción crónica, destaca un predominio de el SBO con un $77 \%$ respecto al DIR 23\%, que es semejante a lo publicado ${ }^{7}$. En cuanto a las características demográficas de los pacientes no encontramos diferencias entre la edad del receptor que en promedio fue de 47 años en SBO y de 46 años en DIR ( $p=0,20)$, y en patología de base en ambos grupos predominó la fibrosis pulmonar idiopática.

Entre los factores de riesgo a considerar para el desarrollo de disfunción crónica de injerto es el grado de sensibilidad a anticuerpos previo al trasplante (PRA) o el desarrollo posterior específico hacia el donante ${ }^{8-9}$. En nuestro estudio no estaban hipersensibilizados y el grupo SBO tenía bajo PRA $<10 \%$ en el $94 \%$ y en grupo DIR $100 \%$ y el desarrollo de nuevos anticuerpos ocurrió en 3 pacientes del grupo SBO y ninguno del grupo DIR.

Los otros factores relacionados al desarrollo de falla de injerto crónica según la literatura son: la disfunción primaria de injerto (DPI) que ocurre en el post operatorio inmediato, episodios de rechazo agudo precoces, la infección a CMV y la asociación con RGE activo ${ }^{10}$. En nuestra revisión la presencia de DPI ocurrió en 6 de 31 pacientes en grupo SBO, 4 de ellas severa, requiriendo soporte de ECMO y ventilación mecánica prolon- 
gada. En el grupo DIR hubo 3 episodios de DPI en 8 pacientes y dos en rango severo.

En relación al rechazo agudo precoz, en el grupo SBO hubo 16 episodios en 14 pacientes y en el grupo DIR 4 episodios en 8 pacientes y el grado histológico predominante fue A 2 (leve) en ambos grupos.

El rechazo humoral o mediado por anticuerpos ocurrió en 2 pacientes con el fenotipo de SBO, no se ha evidenciado este tipo de rechazo en el grupo DIR.

La infección por CMV constituye un problema en los trasplantes de órgano sólido, porque requiere de disminución de inmunosupresión y puede facilitar la disfunción posterior del injerto. Los pacientes con serología positiva al momento del trasplante tienen mayor protección ${ }^{11}$. En nuestra serie SBO hubo $18 \%$ de infección por CMV previa destacando que el $80 \%$ de estos tenían serología negativa a CMV al momento del trasplante. Hubo un solo caso de infección por CMV en grupo DIR.

Otro factor de riesgo mencionado como causa de disfunción crónica de injerto es el RGE activo y esto obliga que los protocolos de estudio previo al trasplante su búsqueda y tratamiento. La presencia de RGE severo no operado o con falla de motilidad de esófago pueden constituir una contraindicación a realizar el trasplante ${ }^{12}$. En nuestro análisis destacó la presencia de RGE activo en $26 \%$ en la serie SBO versus $67 \%$ en la serie DIR $(p=0,02)$ y es importante mencionar que en esta serie hubo 3 pacientes portadores de fibrosis quística avanzada en que la patología esofágica se asocia frecuentemente.

El diagnóstico de disfunción de injerto se realiza por seguimiento espirométrico y habitualmente se considera la caída progresiva de $\mathrm{VEF}_{1}$ sostenida en el tiempo sin otra causa que lo explique ${ }^{13}$. La velocidad del deterioro funcional respiratorio da el pronóstico vital a estos pacientes.

En el grupo SBO de los 31 pacientes, 16 fallecen por esta causa y lo hacen en un tiempo mayor respecto a los que desarrollan DIR, en que se observó rápida declinación espirométrica y de nueve pacientes que fallecieron ocho murieron en un tiempo menor, lo que apoyaría el mal pronóstico expresado en la literatura para este grupo de pacientes $^{3-14}$.

Finalmente podemos concluir que los pacientes trasplantados de pulmón pueden desarrollar diferentes patrones de disfunción crónica de injerto, y aquellos que evolucionan con un patrón restrictivo y aspecto de fibrosis pulmonar en las imágenes tienen un peor pronóstico de vida a mediano plazo.

\section{Bibliografía}

1.- YUSEN R, EDWARDS LB, KUCHERYAVAYA A, BENDEN C, DIPCHAND A, DOBBELS F, et al. The Registry of the International Society for Heart and Lung Transplantation: Thirty-first Adult Lung and Heart-Lung Transplant Report-2014; Focus Theme: Retransplantation. J Heart Lung Transplant 2014; 33 : 1009-24. doi: 10.1016/j.healun.2014.08.004.

2.- VERLEDEN S, SACREAS S, VOS R, VANAUDENAERDE B, VERLEDEN G. Advances in Understanding Bronchiolitis Obliterans after Lung Transplantation. Chest 2016; 150: 219-25.

3.- SATO M, WADDELL T, WAGNETZ U, ROBERTS H, CHAPARRO C, HUTCHEON M, et al. Restrictive Allograft Syndrome (RAS): a novel form of chronic lung allograft dysfunction. J Heart Lung Transplant 2011; 30: 735-42.

4.- VERLEDEN GM, RAGHU G, MEYER KC, GLANVILLE AR, CORRIS P. A new classification system for chronic lung allograft dysfunction. J Heart Lung Transplant 2014; 33: 127-33.

5.- VERLEDEN SE, RUTTENS D, VANDERMEULEN E, BELLON H, VAN RAEMDONCK DE, DUPONT LJ, et al. Restrictive chronic lung allograft dysfunction: where are we now? J Heart Lung Transplant 2015; 34 : 625-30.

6.- VERLEDEN SE, RUTTENS D, VANDERMEULEN E, BELLON H, DUBBELDAM A, DE WEVER W, et al. Predictors of survival in restrictive chronic lung allograft dysfunction after lung transplantation. J Heart Lung Transplant 2016; 35: 1078-84.

7.- KOUTSOKERAA, ROYER P, ANTONIETTI J, FRITZ A, BENDEN C ROUX A, et al. Development of a Multivariate Prediction Model for early-Onset Bronchiolitis Obliterans Syndrome and Restrictive Allograft Syndrome in Lung Transplantation. Frontiers in Medicine 2017; 4: 1-15.

8.- ROYER PJ, OLIVERA-BOTELLO G, KOUTSOKERA A, AUBERT JD, BERNASCONI E, TI SSOT A, et al. Chronic lung allograft dysfunction: a systematic review of mechanisms. Transplantation 2016; 100: 1803-14.

9.- MORRELL MR, PILEWSKI JM, GRIES CJ, PIPELING MR, CRESPO MM, ENSOR CR, et al. De novo donor-specific HLA antibodies are associated with early and high-grade bronchiolitis obliterans syndrome and death after lung transplantation. J Heart Lung Transplant 2014; 33: 1288-94.

10.- VERLEDEN SE, RUTTENS D, VANDERMEULEN E, VANEYLEN A, DUPONT LJ, VAN RAEMDONCK $\mathrm{DE}$, et al. Bronchiolitis obliterans syndrome and restrictive allograft syndrome: do risk factors differ? Transplantation 2013; 95: 1167-72.

11.- VALENTINE VG, GUPTA MR, WALKER JE JR, SEOANE L, BONVILLAIN RW, LOMBARD GA, et al. Effect of etiology and timing of respiratory tract 
infections on development of bronchiolitis obliterans syndrome. J Heart Lung Transplant 2009; 28: 163-9.

12.- MEYER KC, RAGHU G, VERLEDEN GM, CORRIS PA, AURORA P, WILSON KC, et al. An international ISHLT/ATS/ERS clinical practice guideline: diagnosis and management of bronchiolitis obliterans syndrome. Eur Respir J 2014; 44: 1479-503.

13.- LAMA VN, MURRAY S, LONIGRO RJ, TOEWS GB,
CHANG A, LAU C, et al. Course of FEV(1) after onset of bronchiolitis obliterans syndrome in lung transplant recipients. Am J Respir Crit Care Med 2007; 175: 11928.

14.- PISON C, MAGNAN A, BOTTURI K, SEVE M, BROUARD S, MARSLAND BJ, et al. Prediction of chronic lung allograft dysfunction: a systems medicine challenge. Eur Respir J 2014; 43: 689-9.

Correspondencia a:

Dra. María Teresa Parada Correa

Centro de Enfermedades Respiratorias

Clínica Las Condes

Estoril 450 Comuna de Las Condes

Santiago de Chile

Email:mtparada@clc.cl 